\title{
A Numerical Investigation of the Potential for Negative Emissions Leakage
}

\author{
By Niven Winchester and Sebastian Rausch*
}

Leakage of greenhouse gas emissionsincreased emissions in unconstrained regions due to regulations in other regionsundermines the effectiveness of sub-global climate regulations, reduces incentives for unilateral climate initiatives, and can result in distortionary trade measures (Winchester, 2012). These concerns are expressed in measures to reduce leakage included in the EU Emissions Trading Scheme and draft legislation in the US (the now defunct Waxman-Markey bill).

Two sources of leakage include changes in fossil-fuel prices and trade flows (Carbone, Helm and Rutherford, 2009). Leakage via fossil-fossil price effects occurs when reduced energy demand in constrained regions decreases fuel prices and increases fuel use in unconstrained regions. Trade changes contribute to leakage when production increases in unconstrained regions as a result of increased exports to and reduced imports from constrained regions.

Opposing the conventional view, using a theoretical general equilibrium framework, Fullerton, Karney and Baylis (2011) [FKB] show that emissions restrictions may decrease emissions elsewhere due to the abatement resource effect (ARE). The authors assert that negative leakage via the ARE occurs when increased demand for capital and labor to replace fossil fuels in carbon-

\footnotetext{
* Winchester: Joint Program on the Science and Policy of Global Change, Massachusetts Institute of Technology, 77 Massachusetts Avenue, Cambridge, MA 02139 (e-mail: niven@mit.edu); Rausch: Department of Management, Technology and Economics, Zurichbergstrasse 18, 8032 Zurich, Switzerland (e-mail: srausch@ethz.ch). We are grateful to Don Fullerton, Samuel S. Kortum and session participants at the 2013 American Economic Association Annual Meeting for helpful suggestions. The Joint Program on the Science and Policy of Global Change is funded by the US Department of Energy and a consortium of government and industrial sponsors (for the complete list see http://globalchange.mit.edu/sponsors/all).
}

taxed regions attracts factors of production from unregulated regions, which decreases unregulated output and ultimately emissions. ${ }^{1}$

Under the regional interpretation of the model used by FKB, two regions each produce a single good using a "clean" input (a capital and labor composite) and carbon inputs (fossil fuels). The authors impose several general assumptions: (1) the two inputs are imperfect substitutes in production, (2) the two goods are imperfect substitutes in consumption, (3) the clean input is mobile across regions, and (4) the supply of the carbon input is perfectly elastic. As noted by the authors, due to the last assumption, the model excludes leakage due to changes in fossil fuel prices.

Using this framework, the authors relate the change in carbon inputs used in the unconstrained region to a terms-of-trade effect and an ARE. Under the terms-of-trade effect, the higher price of the good produced in the carbon-taxed region induces consumers to substitute towards the good from the other region, which has a positive impact on leakage. As noted earlier, the ARE reduces leakage. Net negative leakage is more likely (i) the lower the elasticity of substitution between the two goods in consumption (as this reduces the termsof-trade effect), and (ii) the higher the elasticity of substitution between the clean and carbon inputs (as this increases the ARE). FKB assert that models that do not allow for inter-regional mobility of capital and labor overestimate leakage, as they omit the ARE.

In the remainder of this paper, in addi-

\footnotetext{
${ }^{1}$ Several authors find negative leakage due to "nonstandard" model extensions, such as endogenous policy responses (see, for example, Copeland and Taylor, 2005). We do not consider such extensions in our analysis.
} 
tion to evaluating the ARE in computable general equilibrium (CGE) models, we extend the framework of FKB in two ways. Specifically, we consider several alternative fossil fuel supply elasticity values, and evaluate the impact of inter-regional capital and labor mobility on leakage. The next section examines leakage using a stylized numerical model, and Section II presents a similar analysis using a multi-region, CGE model of the US economy. Conclusions are summarized in the final section.

\section{A stylized analysis}

We begin by assessing the prospects for negative leakage in a stylized, easily tractable model. The model follows the regional interpretation of FBK's model, with two exceptions. First, to better reflect calibrated numerical general equilibrium models, we specify a home-bias in consumption rather than assuming that all consumers have the same utility function. Second, in addition to considering a case where the supply of carbon inputs is perfectly elastic, we consider several cases where this elasticity is less than infinity.

Our stylized model identifies two symmetric regions ("East" and "West") which each produce a single good. Based on (aggregated) data used for our calibrated general equilibrium model in Section II, we set cost shares for capital-labor $(K)$ and carbon $(C)$ inputs equal to, respectively, 0.98 and 0.02. Goods are trade across regions as imperfect substitutes. In each region, benchmark consumption shares for domestic and foreign goods are equal to, respectively, 0.85 and 0.15 . The equations of the model are set out in the online appendix, which also includes the source code for our numerical simulations.

We investigate the potential for negative leakage by imposing an ad valorem tax of $20 \%$ on carbon inputs in the West and solving the model for alternative values for the elasticity of carbon supply $(\eta)$, and the elasticity of substitution between $K$ and $C$ in the west $\left(\sigma_{\text {West }}^{Y}\right) .^{2}$ Carbon inputs are mo-

\footnotetext{
${ }^{2}$ Changing $\sigma_{W e s t}^{Y}$ is consistent with alternative rep-
}

bile across regions and we implement separate sets of simulations for when $K$ is (i) inter-regionally mobile, and (ii) region specific. To maintain consistency with FKB, changes in the price of $C$ are measured relative to the price of $K$, where the average of prices in the two regions is used for the price of $K$ when this factor is region specific. In our core simulations, we set the elasticity of substitution in production in the East equal to one, and the elasticity of substitution in consumption in both regions equal to 0.75 .

Leakage will occur when the use of $C$ changes in the East. Proportional changes in the this variable when $K$ is mobile across regions are presented in panel (a) of Figure $1 .^{3} \quad$ Leakage is negative for all $\sigma_{\text {West }}^{Y}$ values considered when $\eta=\infty$ and, consistent with $\mathrm{FKB}$, increasing $\sigma_{\text {West }}^{Y}$ reduces leakage. This is because increasing $\sigma_{\text {West }}^{Y}$ induces a larger decrease in demand for $C$ in the West, which ultimately requires a larger decrease in the equilibrium quantity of $C$ supplied to maintain a constant factor price, as illustrated in panel (c) of Figure 1.

When $\eta=0$, the tax simply results in a reallocation of some $C$ inputs from the West to the East and results in positive leakage. Increasing $\sigma_{\text {West }}^{Y}$ allows greater substitution away from $C$ in the West without inducing a larger decrease in supply of this factor so, contrary to when $\eta=\infty$, there is a positive relationship between $\sigma_{\text {West }}^{Y}$ and leakage. For intermediate cases, $0<\eta<\infty$, the tax reduces the equilibrium supply of $C$ but by a smaller amount than when $\eta=\infty$. Consequently, leakage may be positive or negative. In our simulations, for all elasticity combinations, employment of $K$ increases in the West, but leakage is only negative

resentation of advanced, low-carbon technologies, such as renewable electricity generation and electricity from fossil fuels with carbon capture and storage. In unreported simulations, we also vary the substitution elasticities in both regions. Leakage is higher in these simulations than when we only change $\sigma_{W e s t}^{Y}$, as increasing this elasticity in the East allows greater substitution towards fossil fuels in this region.

${ }^{3}$ The climate change literature commonly reports leakage rates, defined as the change in emissions in unconstrained regions divided by the decrease in emissions in regulated regions. Our conclusions for (absolute) leakage derived below also apply to leakage rates. 


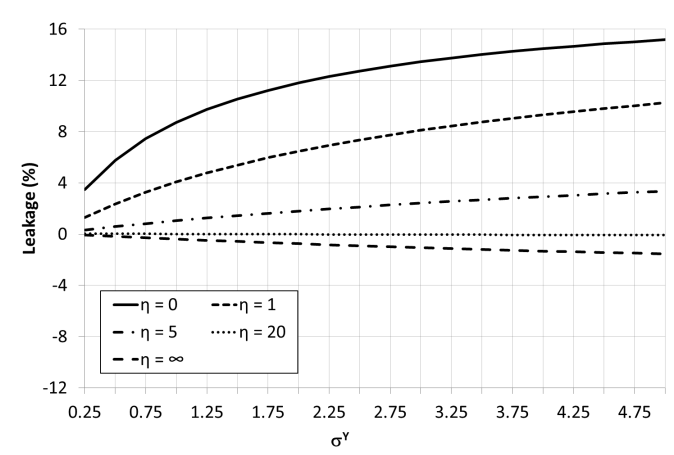

(a) Leakage in East, $K$ mobile across regions.

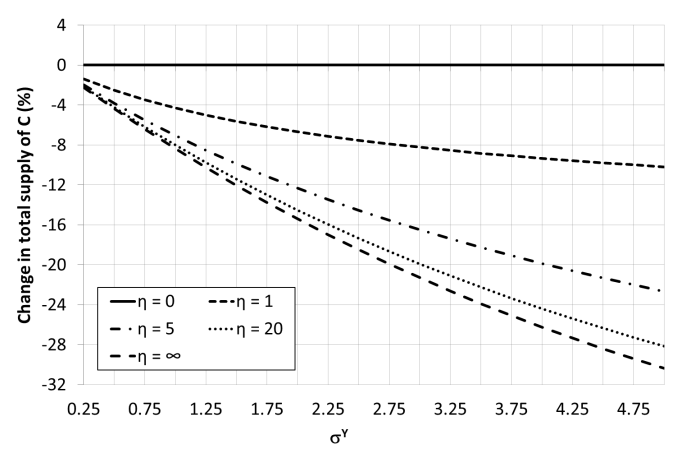

(c) Change in $C$ supply, $K$ mobile across regions.

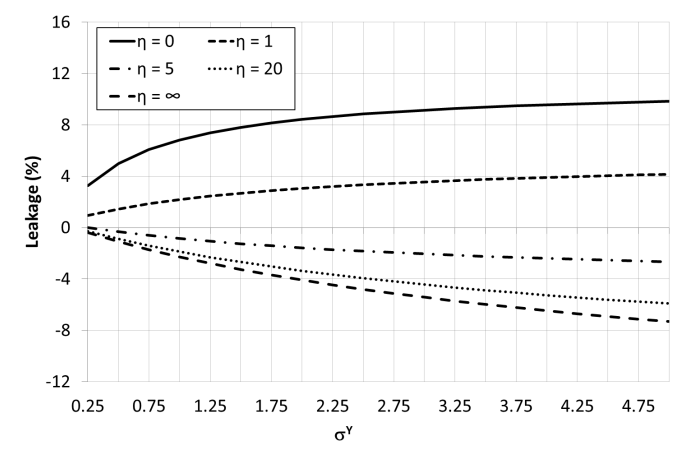

(b) Leakage in East, $K$ immobile across regions.

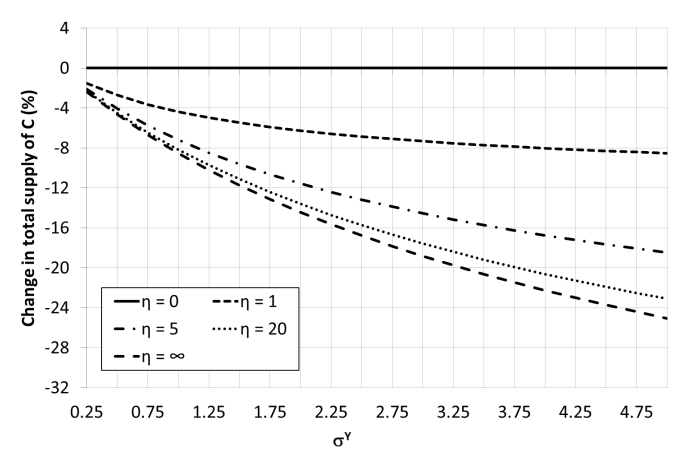

(d) Change in $C$ supply, $K$ immobile across regions.

Figure 1. Leakage And Change in SUPply of CARbon.

for very high values of $\eta$.

Changes in the use of $C$ in the East when $K$ is region-specific are displayed in panel (b) of Figure 1. In comparing results for the two mobility cases, it is helpful to note that allowing $K$ to be mobile across regions has two opposing forces on leakage relative to when this factor is region specific. First, $K$ mobility will result in greater displacement of $C$ in the West, which increases leakage. Second, unless $\eta=0$, the larger reduction of $C$ used in the West requires a larger decrease in the equilibrium supply of $C$ to adhere to the price-quantity relationship specified by the supply elasticity, which decreases leakage.

In our results, for all elasticity combinations, the proportional change in carbon inputs used in the East is smaller when $K$ is region specific than when $K$ is mobile across regions. These results reveal that the displacement effect dominates the supply effect. In other words, in our model, introducing inter-regional mobility of capital and labor in a model that previously specified region-specific factors increases leakage, even though there is a larger decrease in total emissions when $K$ is mobile across regions.

Overall, our results indicate the importance of the supply elasticity for $C$ for observing negative leakage. The intuition behind this result is straight forward: negative leakage can only occur if the decrease in the total equilibrium quantity of $C$ supplied is greater than the reduction in $C$ used in the West. Elasticities of substitution in the production and utility functions affect leakage as they influence the demand for $C$, which interacts with the supply elasticity to determine the equilibrium quantity of $C$. These insights suggest that, in our model, leakage is determined by quantity of $C$ displaced in the constrained region and the impact of this displacement on the total supply of $C$, rather than the constrained region drawing resources away from the unconstrained region as in FKB's theoretical 
analysis.

In sensitivity analyses, we concurrently vary $\eta, \sigma_{\text {West }}^{Y}$, the consumption elasticity, carbon input cost shares, and consumption expenditure shares. Allowing $K$ mobility decreases leakage in only 35 out of 2,700 cases, indicating that our conclusions from our stylized model are robust to the alternative parameterizations considered. Results for alternative consumption elasticities are presented in the online appendix, which also includes the source code to simulate all sensitivity cases. Simulations for which allowing $K$ mobility decreases leakage all involve low values for $\sigma_{\text {West }}^{Y}$ and high values for the elasticity of substitution in consumption.

\section{Analysis using a large-scale CGE model}

We investigate the potential for negative leakage in a large-scale model using a static version of the US Regional Economic Policy (USREP) model described by Rausch et al. (2010). The USREP model is multiregion, multi-sector calibrated general equilibrium model of the US economy with detailed representation of energy extraction and production that is benchmarked to 2006 data. The model is built on statelevel input-output and trade data from IMPLAN (2008), and state-level data on energy balances and prices from EIA (2009). Using a model of sub-federal economies allows us to examine leakage due to a subfederal policy, which we prefer to a national climate initiative as capital and labor are more mobile within nations than across international borders.

We aggregate the data to identify five regions based on US Census Bureau groupings: West, Midwest, Northeast, South Atlantic, and South Central. Our sectoral aggregation includes five energy sectors (Coal, Crude oil, Gas, Refined oil, and Electricity) and five non-energy sectors (Agriculture, Energy-intensive industry, Other industry, Transportation, and Services).

Crude oil is a homogenous commodity in the model. For other commodities, the model tracks bilateral trade among US re- gions and, following Armington (1969), assumes that imports are differentiated by region of origin. Operationalizing our import specification requires assigning values for elasticities of substitution between imports from different regions, and between aggregate imports and domestic production (trade elasticities), which we source from Beckman, Hertel and Tyner (2011) and Caron, Rausch and Winchester (2012).

We model the foreign sector by endowing each region with a exogenous quantity of foreign imports and requiring each region to produce a fixed quantity of international exports. ${ }^{4}$ We also assume that all regions face a fixed price of crude oil. These assumptions eliminate leakage to foreign regions and allow us to focus on sub-national leakage.

The model identifies five production factors: capital, labour, and sector-specific resources for Coal, Crude oil and Gas. Production in each sector combines intermediate inputs and factors of production using nested constant elasticity of substitution (CES) functions. The utility function for each region is also a series of nested CES functions of commodities entering final demand. Key drivers of abatement possibilities include trade elasticities and the elasticity of substitution between aggregate energy and capital-labor $\left(\sigma^{Y}\right)$ in production, especial in the electricity sector.

Fossil fuel $f$ is produced according to a nested CES function combining a fuelspecific resource, $R$, and non-resource inputs (comprising capital, labor, and intermediate inputs), $V$ :

$$
Y_{f}=\left[\alpha_{f} R_{f}^{\rho_{f}}+\left(1-\alpha_{f}\right) V_{f}^{\rho_{f}}\right]^{1 / \rho_{f}}
$$

where $Y, \alpha, \sigma_{f}=1 /\left(1-\rho_{f}\right)$ is output, the share coefficients of the CES function, and the elasticity of substitution between the resource and non-resource inputs, respectively. Given the form of the production function in Eq. (1), the elasticity of substitution between the resource and the rest of inputs in the top nest determines the

\footnotetext{
${ }^{4}$ This representation is similar to that used by Goulder, Hafstead and Dworsky (2010).
} 
price elasticity of supply $\left(\eta_{f}\right)$ at the reference point according to: ${ }^{5}$

$$
\eta_{f}=\sigma_{f} \frac{1-\alpha_{f}}{\alpha_{f}} .
$$

Large-scale applied CGE models typically employ fuel supply elasticities for coal and natural gas ranging from, respectively, 0.8-1.2 and 0.5-0.8 (see, for example, the EPPA model, Paltsev et al., 2005; the GTAP model, Beckman, Hertel and Tyner, 2011; the USREP model, Rausch et al., 2010; and CIM-EARTH, Elliott et al., 2010). These supply elasticities typically imply elasticities of substitution for coal and natural gas of about 0.7 and 0.6 , respectively.

Using the USREP model, we implement a carbon tax of $\$ 30$ per metric ton of carbon dioxide $\left(\mathrm{tCO}_{2}\right)$ in the West. Reflecting regional electricity markets, electricity is not traded between the West and other regions in our model, so our leakage calculations are not driven by changes in electricity trade. As for our stylized analysis, we simulate our policy scenario under two alternative model specifications: one with regionspecific capital and labor (which does not allow for the ARE), and one with labor and capital that is perfectly mobile across regions (which does allow for the ARE). For each specification, we consider alternative values for $\sigma^{Y}$ in the West and trade elasticities in all regions. ${ }^{6}$

Proportional changes in $\mathrm{CO}_{2}$ emissions aggregated across unconstrained regions are reported in Figure 2. Overall, the results mirror those from the stylized analysis. There is a strong positive relationship between leakage and the supply elasticity for fossil fuels. Increasing $\sigma^{Y}$ may increase or decrease leakage. Allowing inter-regional capital and labor mobility increases leakage relative to when these factors are region

\footnotetext{
${ }^{5}$ For the derivation of the relationship between $\eta, \alpha$, and $\sigma$, see Rutherford $(2002$, p. 20$)$.

${ }^{6}$ As noted in Section I, increasing $\sigma^{Y}$ in the West allows us to consider abatement opportunities due to the availability of advanced technologies. An alternative approach is to explicitly model advanced technologies. To maintain consistency with the theoretical framework of FKB, we prefer to vary the value of $\sigma^{Y}$
}

specific, except for a small number of cases with high $\sigma^{Y}$ values. One difference is that leakage is always positive for all elasticity combinations, both in the results presented in Figure 2 and results from a detailed sensitivity analysis. ${ }^{7}$ These results indicate that there is little potential for negative leakage in calibrated general equilibrium models based on real-world data.

\section{Conclusion}

This paper investigated the potential for negative leakage across regions in CGE models due to the ARE identified by FKB. Analysis using a stylized model illustrated two important relationships. First, leakage is determined by the interaction of the elasticities of substitution in the production and utility functions, which influence the demand for carbon inputs, and the supply elasticity for the carbon inputs. Second, in our stylized model, allowing inter-region mobility of capital and labor increased leakage, as more carbon inputs are displaced in the carbon constrained region relative to when capital and labor are region specific. These findings indicate that models with region-specific production factors do not overestimate leakage.

Using a multi-region model of the US, we found that allowing inter-regional mobility of capital and labor had little impact on leakage. Also, leakage was positive for all parameterizations we considered. We conclude that there is little prospect for negative leakage in conventional numerical general equilibrium models. A key reason why leakage is positive is that numerical general equilibrium models are calibrated to fossil fuel supply elasticity values less than one, rather than the very high elasticity values required to generate negative leakage in our stylized model and low leakage rates in the USREP model.

\section{REFERENCES}

Armington, Paul. 1969. "A Theory of

\footnotetext{
${ }^{7}$ In the online appendix, we report results for "low" and "high" values for trade elasticities in all regions, where low and high values are equal to base values multiplied by, respectively, 0.5 and 2 .
} 


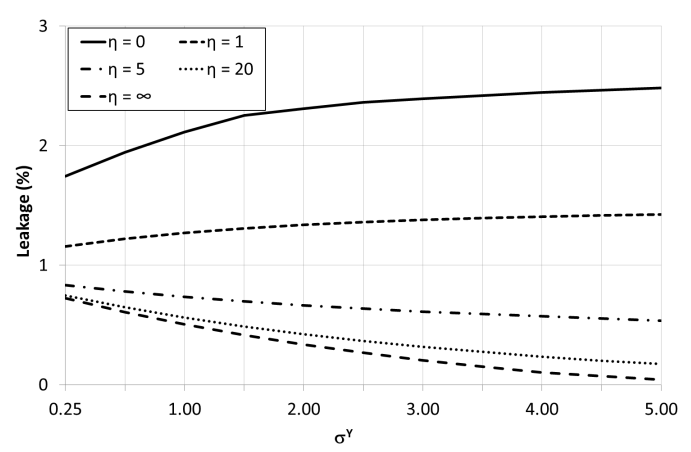

(a) Full mobility of capital and labor across regions.

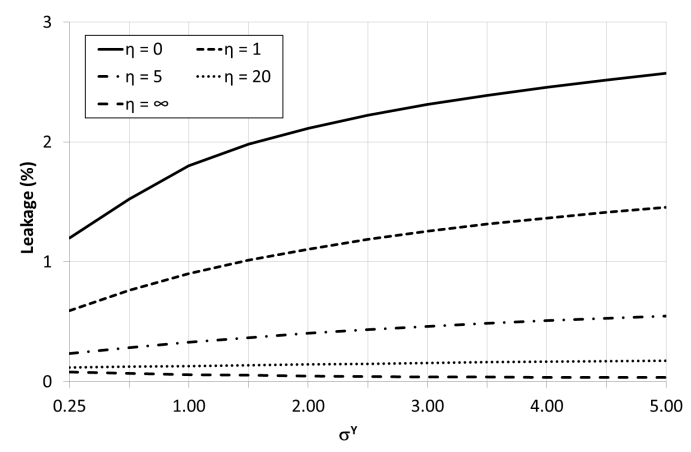

(b) No mobility of capital and labor across regions.

Figure 2. Leakage to unconstrained U.S. Regions.

Demand for Products Distinguished by Place of Production." International Monetary Fund Staff Papers, 16: 159-76.

Beckman, J., T. Hertel, and W. Tyner. 2011. "Validating Energyoriented CGE Models." Energy Economics, 33(5): 799-806.

Carbone, Jared C., Carsten Helm, and Thomas F. Rutherford. 2009. "The Case for International Emission Trade in the Absence of Cooperative Climate Policy." Journal of Environmental Economics and Management, 58(3): 266280.

Caron, Justin, Sebastian Rausch, and Niven Winchester. 2012. "Leakage from Sub-national Climate Initiatives: The Case of California." MIT Joint Program on the Science and Policy of Global Change, Report No. 220,

Copeland, Brian R., and M. Scott Taylor. 2005. "Free Trade and Global Warming: a Trade Theory View of the Kyoto Protocol." Journal of Environmental Economics and Management, 205-234(49): 2.

Dirkse, S. P., and M. C. Ferris. 1995. "The PATH Solver: a Non-monontone Stabilization Scheme for Mixed Complementarity Problems." Optimization Methods and Software, 5: 123-156.
EIA, (Energy Information Administration). 2009. "State Energy Data System." Washington, DC.

Elliott, Joshua, Ian Foster, Kenneth Judd, Elisabeth Moyer, and Todd Munson. 2010. "CIMEARTH: Community Integrated Model of Economic and Resource Trajectories for Humankind, v1.0." Argonne National Laboratory. Available at: http://www.cimearth.org/files/cimearthv01.pdf.

Fullerton, Don, Dan Karney, and Kathy Baylis. 2011. "Negative Leakage." CESinfo Working Paper No. 3379.

Goulder, Lawrence H., Marc A.C. Hafstead, and Michael Dworsky. 2010. "Impacts of Alternative Emissions Allowance Allocation Methods under a Federal Cap-and-trade Program." Journal of Environmental Economics and Management, 60(3): 161-181.

IMPLAN. 2008. "State-Level U.S. Data for 2006." Stillwater, MN: Minnesota IMPLAN Group.

Mathiesen, Lars. 1985. "Computation of Economic Equilibria by a Sequence of Linear Complementarity Problems." Mathematical Programming Study, 23: $144-162$.

Paltsev, Sergey, John M. Reilly, Henry Jacoby, Richard Eckhaus, Jim McFarland, Marcus Sarofim, 
M. Asadoorian, and Mustafa Babiker. 2005. "The MIT Emissions Prediction and Policy Analysis (EPPA) Model: Version 4." MIT Joint Program on the Science and Policy of Global Change, Report 125, Cambridge, MA.

Rausch, Sebastian, Gilbert E. Metcalf, John M. Reilly, and Sergey Paltsev. 2010. "Distributional Implications of Alternative U.S. Greenhouse Gas Control Measures." The B.E. Journal of Economic Analysis $\& 3$ Policy, 10(2). Symposium.

Rutherford, Thomas F. 1995. "Extension of GAMS for Complementarity Problems arising in Applied Economics." Journal of Economic Dynamics and Control, 19(8): 1299-1324.

Rutherford, Thomas F. 2002. "Lecture Notes on Constant Elasticity Functions." Unpublished manuscript. University of Colorado. Available at: http://www.gamsworld.eu/mpsge/debre u/ces.pdf.

Winchester, Niven. 2012. "The Impact of Border Carbon Adjustments under Alternative Producer Responses." American Journal of Agricultural Economics, 94(2): 354-359.

\section{Appendix A: Equilibrium Conditions for Stylized Model used in Section I}

Our stylized general equilibrium model is modified version of the regional interpretation of the model presented in Fullerton, Karney and Baylis (2011). Two regions $(r=\{$ East, West $\})$ produce a good $Y$ combining two inputs indexed by $f=\{K, C\}$ with decreasing marginal products in a constant returns to scale production function. Let $K$ denote the clean input, and $C$ represents carbon inputs. The clean input can be considered to be a composite of labor and capital, and is assumed to be in fixed supply. We distinguish two cases: one in which the clean input is mobile and one in which it is immobile across regions. In response to a carbon tax, a firm can reduce its carbon per unit of output by additional use of abatement technology, that is by substituting from $C$ into $K$. $Y$ can be traded at no costs, and demand for $Y$ in each region is derived from maximizing homothetic utility by choice of $Y_{\text {East }}$ and $Y_{\text {West }}$ subject to an income constraint, taking as given all market prices. We compare the long run equilibrium after imposing a tax on carbon inputs in the West, ignoring adjustments during the transition. We solve the model for alternative values of the elasticity of supply for carbon inputs, including the perfectly elastic case considered by FKB.

We now present the equilibrium conditions of the generalized FKB model that is used to derive the results from the first part of the paper. We employ numerical methods to solve for general equilibrium prices and quantities. More specifically, we formulate the model as a system of nonlinear inequalities and represent the economic equilibrium through two classes of conditions: zero profit and market clearance. The former class determines activity levels and the latter determines price levels. In equilibrium, each of these variables is linked to one inequality condition: an activity level to an exhaustion of product constraint and a commodity price to a market clearance condition. Following Mathiesen (1985) and Rutherford (1995), we formulate the model as a mixed complementarity problem. ${ }^{8}$

In equilibrium, activity levels for $Y_{r}$ are determined by the following set of zeroprofit conditions:

$$
\Pi_{r}^{Y}=p_{r}^{Y}-\left(\sum_{f} \theta_{f, r}^{Y} w_{f, r}^{1-\sigma_{r}^{Y}}\right)^{1 /\left(1-\sigma_{r}^{Y}\right)} \leq 0
$$

\footnotetext{
${ }^{8} \mathrm{~A}$ complementary-based approach has been shown to be convenient, robust, and efficient (Mathiesen, 1985; Rutherford, 1995). A characteristic of many economic models is that they can be cast as a complementary problem, i.e. given a function $F: \mathbb{R}^{n} \longrightarrow \mathbb{R}^{n}$, find $z \in \mathbb{R}^{n}$ such that $F(z) \geq 0, z \geq 0$, and $z^{T} F(z)=0$, or, in short-hand notation, $F(z) \geq 0 \perp z \geq 0$. The complementarity format embodies weak inequalities and complementary slackness, relevant features for models that contain bounds on specific variables, e.g. activity levels which cannot a priori be assumed to operate at positive intensity. Numerically, we solve the model in GAMS using the PATH solver (Dirkse and Ferris, 1995).
} 


$$
\perp \quad Y_{r} \geq 0
$$

where $p_{r}^{Y}, \theta_{f, r}^{Y}, w_{K, r}$ denote output price indexes, the benchmark value share, and the price for the clean input, respectively. $\sigma_{r}^{Y}$ represents the elasticity of substitution between $K$ and $C$, i.e. a measure of the technological ease with which to abate carbon emissions, everything else equal. Let $n_{r}$ denote the price for the carbon input "carbon emissions". The price gross of the carbon $\operatorname{tax} \tau_{r}, w_{C, r}$ is then given by:

$$
w_{C, r}=n_{r}\left(1+\tau_{r}\right) \quad \perp \quad w_{C, r} \geq 0 .
$$

We consider the following case: $\tau_{\text {West }}>0$ and $\tau_{\text {East }}=0$.

Utility in each region is "produced" by combining locally-produced and imported varieties of $Y$. The level of utility is determined in equilibrium by the following conditions:

$$
\Pi_{r}^{U}=p_{r}^{U}-\left(\sum_{r^{\prime}} \theta_{r^{\prime}}^{U} p^{Y} r^{1-\sigma^{C}}\right)^{1 /\left(1-\sigma^{C}\right)} \leq 0
$$

$$
\perp \quad U_{r} \geq 0
$$

where $\sigma^{C}$ represents the elasticity of substitution between locally-produced and imported varieties, and $\theta_{r}^{U}$ denote the respective value shares.

Assuming that the revenue from the carbon tax is returned lump-sum, the income of the representative consumer in each region is given by:

$$
M_{r}=w_{K, r} \bar{E}_{r}+n_{r}\left(1+\tau_{r}\right) \zeta_{r}
$$

where $\bar{E}_{r}$ denotes the fixed supply of $K$ and $\zeta_{r}$ is the supply of the carbon input, which is determined endogenously by targeting the price elasticity of fossil fuel supply, $\eta .^{9}$ If $\eta<\infty$, then this condition is given by:

$$
\frac{\zeta_{r}-1}{n_{r}-1}=\eta \quad \perp \quad \zeta_{r} \geq 0 .
$$

If the supply of fossil fuels is perfectly elas-

\footnotetext{
${ }^{9}$ Note that this formulation assumes a constant elasticity supply function.
}

tic, i.e. $\eta=\infty$, then:

$$
n_{r}=1 \quad \perp \quad \zeta_{r} \geq 0 .
$$

Note that we calibrate the model such that initially all prices and quantities are equal to one. Hence, in Eq. (7) the left-hand side is simply the percentage change in the quantity of fossil fuel supplied over the percentage change in the price for fossil fuel, i.e. the price elasticity of supply.

Using Shephard's Lemma, differentiating the unit profit function with respect to input prices provides compensated demand coefficients, which appear subsequently in the market clearance conditions. The equilibrium price for clean inputs is determined by:

$$
\bar{E}_{r} \geq \frac{\partial \Pi_{r}^{Y}}{\partial w_{K, r}} Y_{r} \quad \perp \quad w_{K, r} \geq 0 .
$$

The following condition determines the equilibrium price for carbon inputs:

$$
\bar{E}_{C, r} \zeta_{r} \geq \frac{\partial \Pi_{r}^{Y}}{\partial n_{r}} Y_{r} \quad \perp \quad n_{r} \geq 0 .
$$

Locally non-satiated preferences imply that all income is exhausted, and hence the market for utility clears if:

$$
U_{r} \geq \frac{M_{r}}{p_{r}^{U}} \quad \perp \quad p_{r}^{U} \geq 0 .
$$

In the equations above, we have represented the case where the clean input is immobile across regions. We also consider the case where $K$ is mobile across regions, hence implying a uniform rental price for $K$, $w_{K}$. In this version of the model, Eq. (9) is replaced by:

$$
\sum_{r} \bar{E}_{r} \geq \sum_{r} \frac{\partial \Pi_{r}^{Y}}{\partial w_{K}} Y_{r} \quad \perp \quad w_{K} \geq 0 .
$$

and $w_{K, r}$ in equations (3) and (6) is replaced by $w_{K}$. 


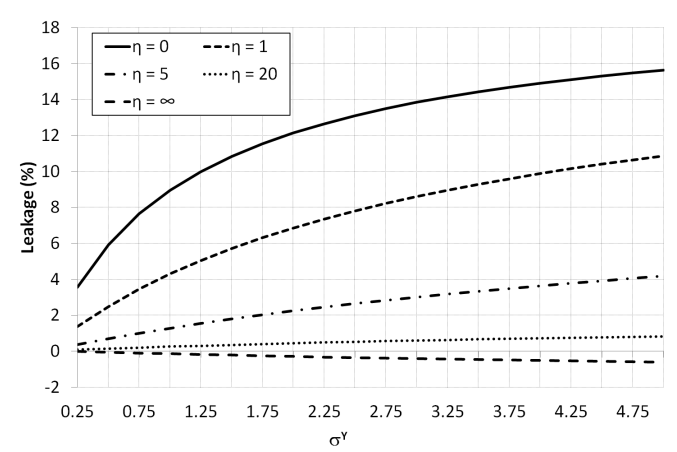

(a) Full factor mobility, base case consumption elasticity.

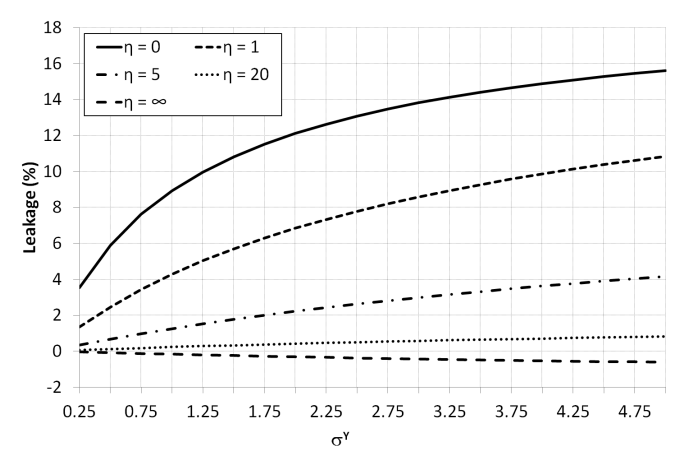

(c) Full factor mobility, low consumption elasticity.

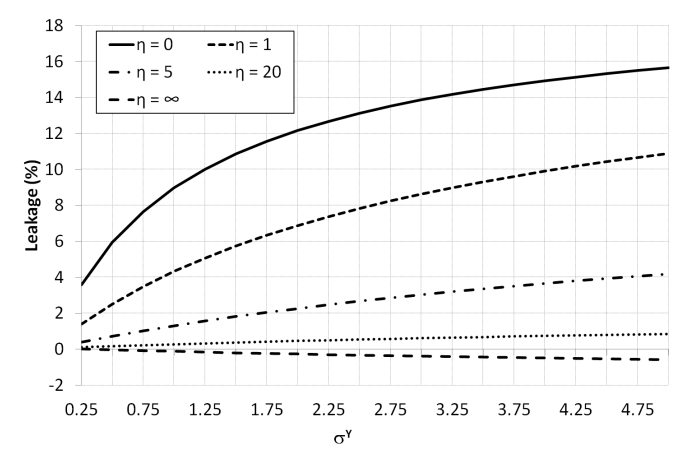

(e) Full factor mobility, high consumption elasticity.

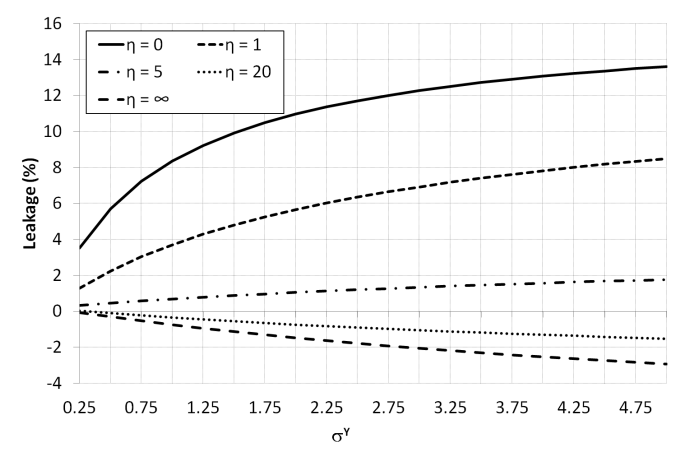

(b) No factor mobility, base case consumption elasticity.

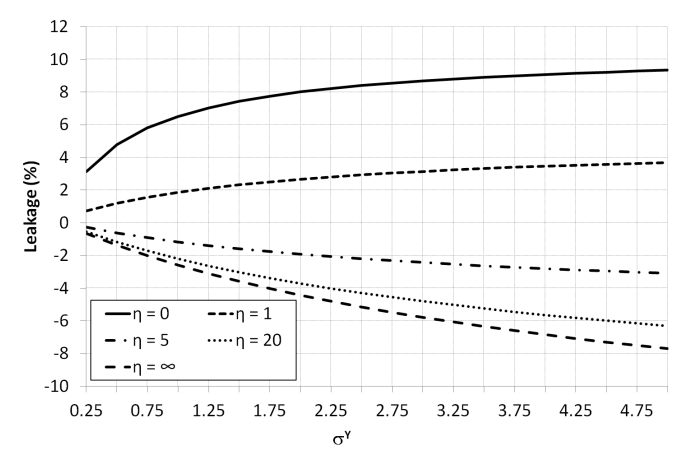

(d) No factor mobility, low consumption elasticity.

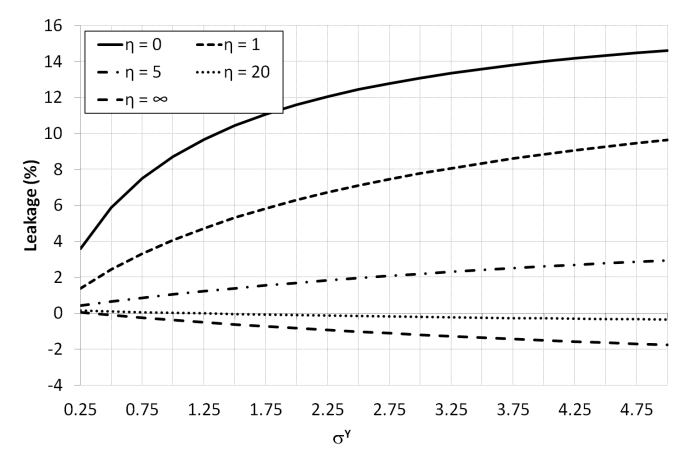

(f) No factor mobility, high consumption elasticity.

Figure 3. Stylized GE model: Leakage for different values for consumption elasticity (\%) 


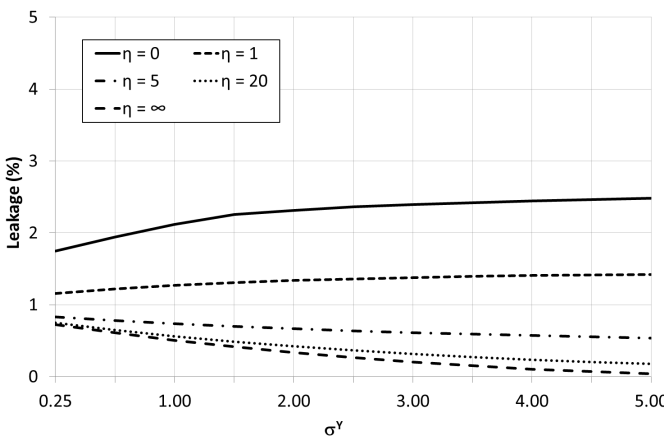

(a) Full factor mobility, base case trade elasticities.

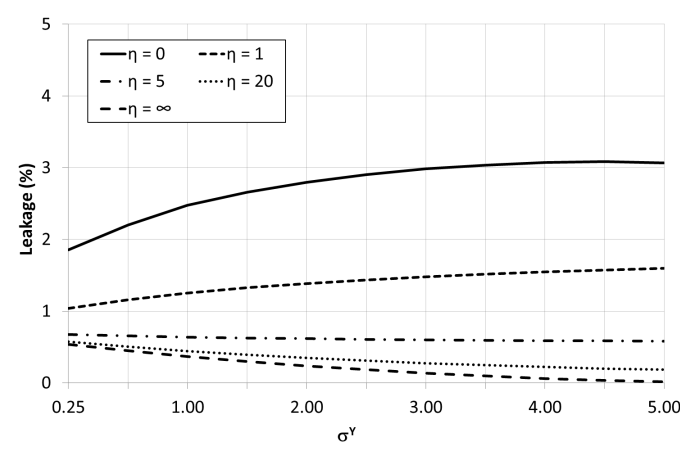

(c) Full factor mobility, low trade elasticities.

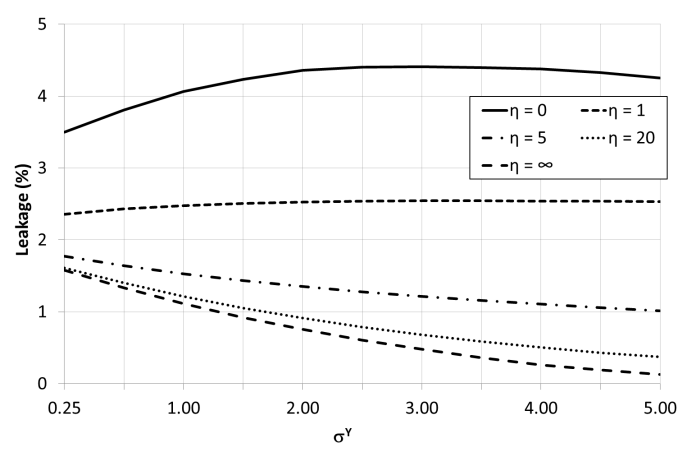

(e) Full factor mobility, high trade elasticities.

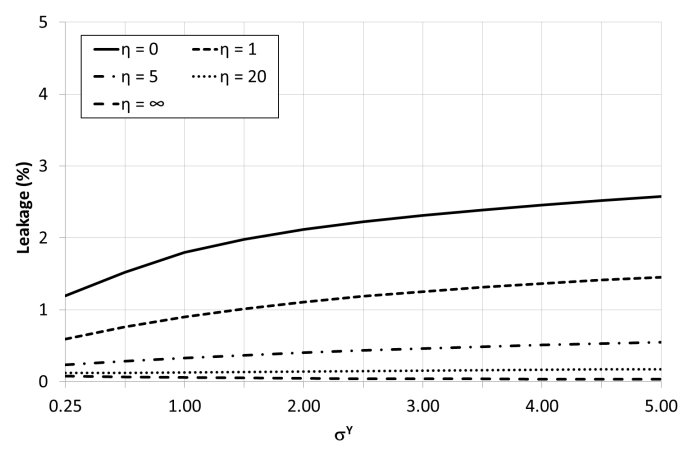

(b) No factor mobility, base case trade elasticities.
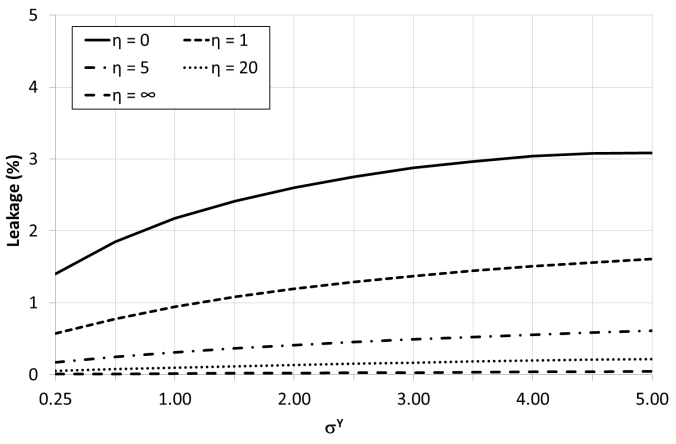

(d) No factor mobility, low trade elasticities.

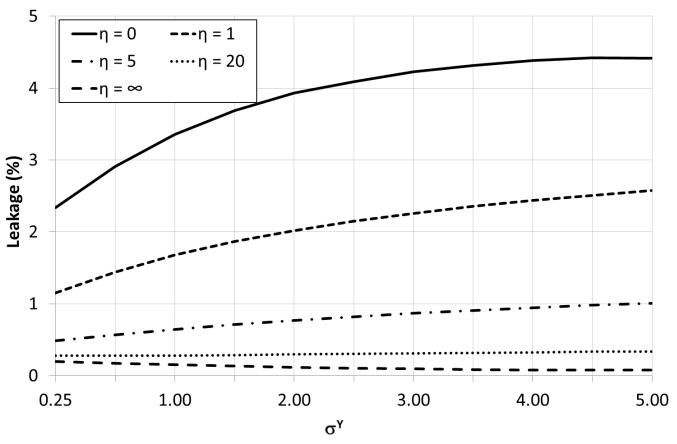

(f) No factor mobility, high trade elasticities.

Figure 4. USREP model: Leakage for alternative trade elasticity Values (\%) 
Appendix B: Computer code for stylized model using GAMS and GAMS/MPSGE

\$TITLE A stylized GE model to examine the impact of a carbon tax on negative leakage * Sets and parameters:

SET

f Factors of production /carb,kl/,

$r$ Regions/east,west/;

$\operatorname{ALIAS}(r, r r)$;

* Set a scalars to control input using a .bat file:

SCALAR m Carbon mobility indicator

s Carbon factor payments

a Expenditure on the domestic good;

$\mathrm{m}=\% \mathrm{~m} \% ; \mathrm{s}=\% \mathrm{~s} \% ; \quad \mathrm{a}=\% \mathrm{a} \%$;

PARAMETER

$\operatorname{vom}(r)$ Value of output

$\operatorname{vfm}(f, r)$ Value of factor input

$\mathrm{vdm}(\mathrm{r}, \mathrm{rr})$ Consumption of good produced in $\mathrm{rr}$ consumed in region $r$ tf $(f, r)$ Ad valorem factor tax

$\operatorname{esubp}(r)$ Elasticity of substitution in production

esubc Elasticity of subtitution in consumption

eta Own-price elasticity of supply for fossil fuels

fcarb(f) Flag for endogenous carbon mutilpier

mobile(f) Flag for factor mobility;

* Assign production and consumption values:

$\operatorname{vfm}(" \mathrm{carb} "$, "east") = s;

$\operatorname{vfm}("$ carb", "west") = s;

$\operatorname{vfm}(" k l ", r)=100-\operatorname{vfm}(" \operatorname{carb} ", r)$;

$\operatorname{vom}("$ east" $)=\operatorname{sum}(f, \operatorname{vfm}(f$, "east") $)$;

$\operatorname{vom}(" w e s t ")=\operatorname{sum}(f, \operatorname{vfm}(f$, "west") $)$;

$\operatorname{vdm}(r, r)=a$;

vdm("east", "west") $=100-\operatorname{vdm}($ "east", "east");

vdm("west", "east") = 100 - vdm("west", "west");

* Set initial values for elasticities and flags:

tf $(f, r)=0$;

$\operatorname{esubp}(r)=1$;

esubc $=0.5$

eta $=0$;

$f \operatorname{carb}(f)=$ no;

mobile ("carb") = 1 ;

mobile ("kl") = m; 
* Declare general equilibrium model using GAMS/MPSGE.

* References for GAMS and MPSGE subsystem:

* Rutherford, Thomas F. 1995. Extension of GAMS for Complementarity Problems

* arising in Applied Economics. Journal of Economic Dynamics and Control, * $19(8)$ : 12991324.

* Rutherford, Thomas F. 1999. Applied General Equilibrium Modeling with MPSGE

* as a GAMS Subsystem: an Overview of the Modeling Framework and

* Syntax. Computational Economics, 14: 146.

\$ONTEXT

\$MODEL : LEAKAGE

\$SECTORS :

$\mathrm{Y}(\mathrm{r})$ ! Output index

W(r) ! Welfare index

$\mathrm{Z}(\mathrm{f}, \mathrm{r})$ ! Dummy factor production block

\$COMMODITIES :

$P Y(r)$ ! Output price index

$P F(f) \$($ mobile $(f)$ eq 1) ! Factor price index - regionally mobile factors

$\operatorname{PFF}(f, r) \$($ mobile $(f)$ eq 0$)$ ! Factor price index - region specific factors

$\mathrm{PZ}(f, r)$ ! Factor price index

PW(r) ! Price index for welfare

\$CONSUMERS :

$\mathrm{HH}(r)$ ! Household income and expenditure

\$AUXILIARY:

TAU(f)\$fcarb(f) ! Carbon endowment multiplier

\$PROD:Y(r) s:esubp $(r)$

$0: P Y(r) Q: \operatorname{vom}(r)$

$I: P Z(f, r) \quad Q: v f m(f, r)$

\$PROD : Z $(f, r)$

$0: P Z(f, r) \quad Q: v f m(f, r)$

$I: P F(f) \$(\operatorname{mobile}(f)$ eq 1) $Q: v f m(f, r) A: H H(r)$ T:tf $(f, r)$

$I: \operatorname{PFF}(f, r) \$($ mobile $(f)$ eq 0$) \quad Q: v f m(f, r) A: H H(r) \quad T: t f(f, r)$

\$PROD:W(r) s:esubc

$0: P W(r) Q:(\operatorname{sum}(r r, v d m(r, r r)))$

$I: P Y(r r) \quad Q: \operatorname{vdm}(r, r r)$

\$DEMAND : $\mathrm{HH}(r)$

$D: P W(r) \quad Q:(\operatorname{sum}(r r, \operatorname{vdm}(r, r r)))$

$E: P F(f) \$($ mobile (f) eq 1) $Q: \operatorname{vfm}(f, r) r: T A U(f) \$ f \operatorname{carb}(f)$

$E: \operatorname{PFF}(f, r) \$(\operatorname{mobile}(f)$ eq 0$) \quad Q: \operatorname{vfm}(f, r) r: T A U(f) \$ f \operatorname{carb}(f)$

\$REPORT :

$\mathrm{V}: \operatorname{OUTPUT}(r) \quad 0: \mathrm{PY}(r)$ PROD:Y(r)

$\mathrm{V}: \operatorname{INPUT}(f, r) \quad I: P Z(f, r)$ PROD $: Y(r)$ 
\$CONSTRAINT:TAU( $f) \$(f \operatorname{carb}(f)$ and eta lt inf)

* Express the price of carb relative to the average price of $k l$

$(\operatorname{tau}(f)-1) /((p f(f) /(0.5 * P Z(" k l "$, "west" $)+0.5 * P Z(" k l "$, "east" $)))-1)=e=$ eta;

\$CONSTRAINT:TAU( $f) \$(f \operatorname{carb}(f)$ and eta eq inf)

* Express the price of carb relative to the average price of $\mathrm{kl}$

$\mathrm{PF}($ "carb") $=\mathrm{e}=0.5 * \mathrm{PZ}(" \mathrm{kl}$ ", "west") $+0.5 * \mathrm{PZ}(" \mathrm{kl}$ ", "east");

\$OFFTEXT

\$SYSINCLUDE mpsgeset LEAKAGE

* Set the initial value of the carbon endowment multiplier:

TAU.L $(f)=1$;

* Check the benchmark:

LEAKAGE. iterlim = 0;

\$INCLUDE LEAKAGE.GEN

SOLVE LEAKAGE USING MCP;

LEAKAGE. iterlim $=10000$;

\$INCLUDE LEAKAGE.GEN

SOLVE LEAKAGE USING MCP;

* Impose a carbon tax or a cap-and-trade policy:

tf $($ "carb", "west") $=0.2$;

\$INCLUDE LEAKAGE.GEN

SOLVE LEAKAGE USING MCP;

* Allow non-zero supply elasticity responses:

fcarb $(" c a r b ")=$ yes;

eta $=1$;

\$INCLUDE LEAKAGE.GEN

SOLVE LEAKAGE USING MCP;

* Loop over alternative parameter values:

SET p Prroduction elasticity values /esubp025, esubp05, esubp75, esubp100, esubp125, esubp150, esubp175, esubp200, esubp225, esubp250, esubp275, esubp300, esubp325, esubp350, esubp375, esubp400, esubp425, esubp450, esubp475, esubp500/;

SET c Consumption elasticity values /esubc05, esubc75, esubc10/;

SET e Fossil fuel supply elasticities /eta0, eta1, eta5, eta20, etainf/;

* Extract results of interest:

PARAMETER

Input_loop( $f, r, p, c, e)$ Percentage change in input

fesubp(r) Flag to change the production elasticity

carb_east(p,c,e) Percentage change in carbon inputs employed in the East 


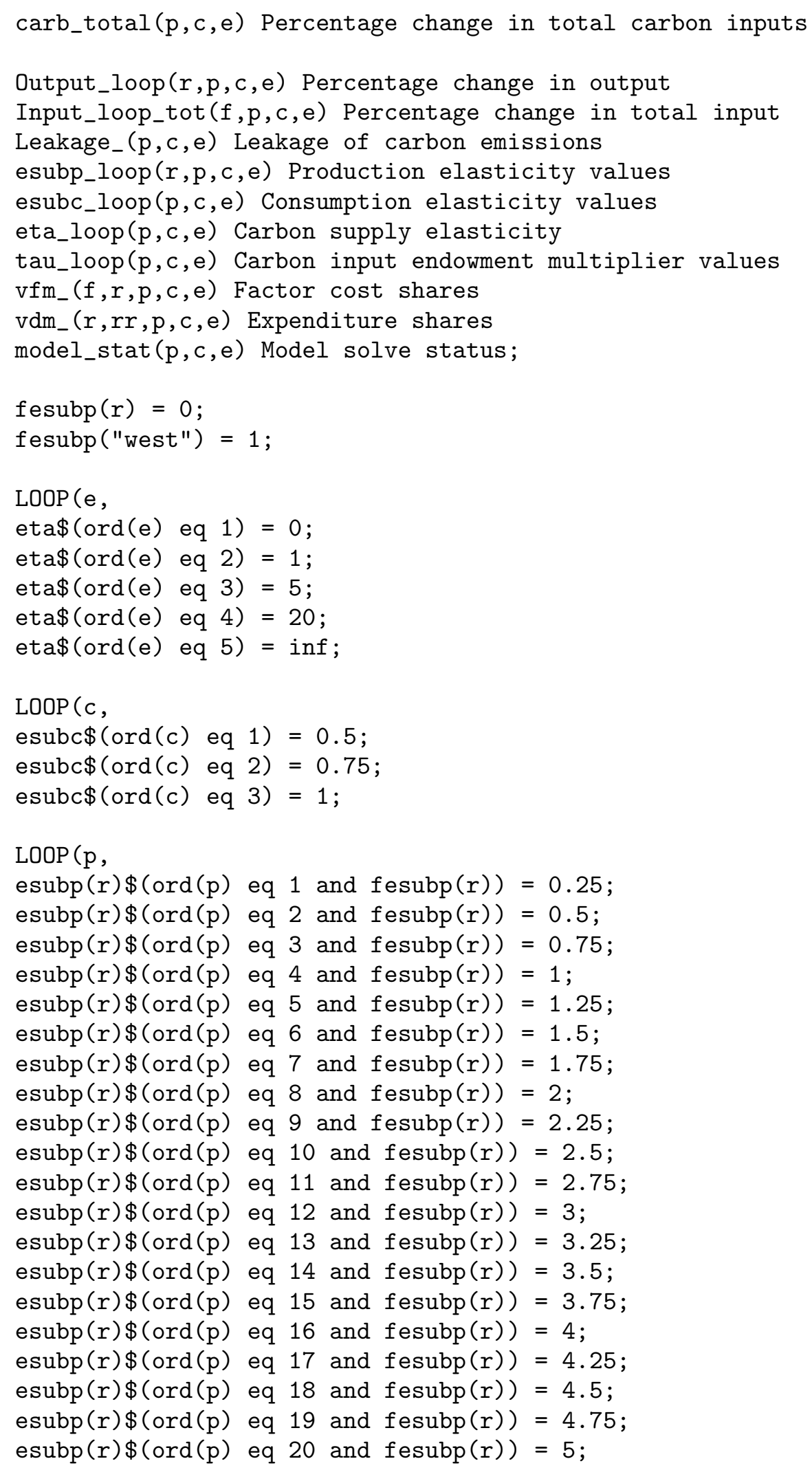




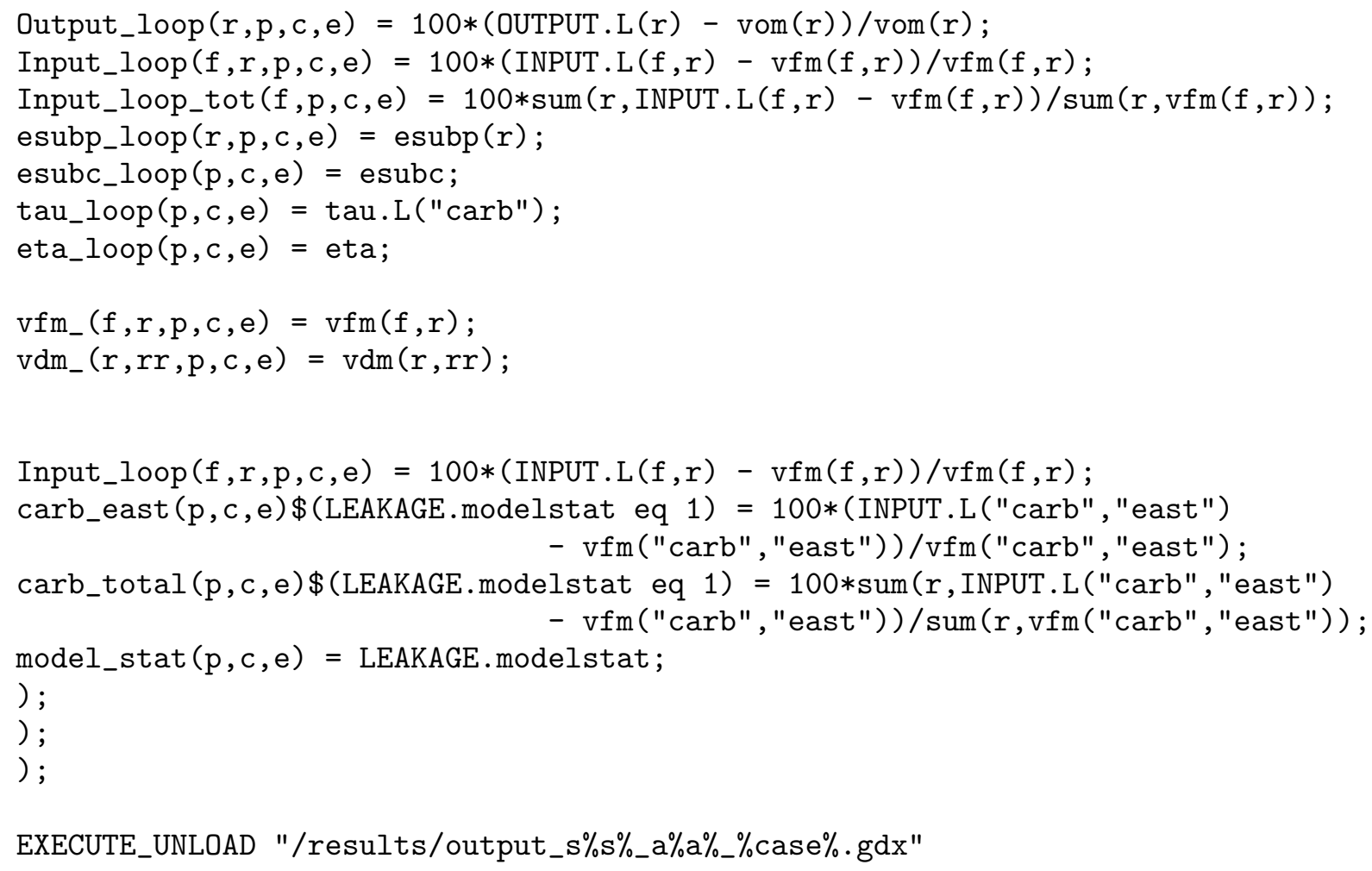

